\title{
Enzymatic Conversion of Odorants in Nasal Mucus Affects Olfactory Glomerular Activation Patterns and Odor Perception
}

\author{
Ayumi Nagashima and Kazushige Touhara \\ Department of Applied Biological Chemistry, The University of Tokyo, Tokyo 113-8675, Japan
}

Odor information is decoded by a combination of odorant receptors, and thus transformed into discrete spatial patterns of olfactory glomerular activity. It has been found, however, that for some odorants, there are differences between the ligand specificity of an odorant receptor in vitro and its corresponding glomerulus in vivo. These observations led us to hypothesize that there exist prereceptor events that affect the local concentration of a given odorant in the nasal mucus, thus causing the apparent specificity differences. Here we show that odorants with functional groups such as aldehydes and esters are targets of metabolic enzymes secreted in the mouse mucus, resulting in their conversion to the corresponding acids and alcohols. The glomerular activation patterns elicited by an enzyme-targeted odorant in the olfactory bulb was different in the presence of an enzyme inhibitor in the mucosa, suggesting that the enzymatic conversion occurs fast enough to affect recognition of the odorant at the levels of olfactory sensory neurons. Importantly, olfactory discrimination tests revealed that mice behaviorally trained to associate an enzyme-targeted odorant to sugar rewards could not discriminate the odorant after treatment with the enzyme inhibitor. These results reveal that the enzymatic conversion of odorants in the nasal mucus appears be fast enough to affect olfactory perception, which sheds light on the previously unappreciated role of nasal mucosal enzymes in odor sensation.

\section{Introduction}

The mammalian olfactory system can detect and discriminate numerous volatile odorous compounds. Odorants are recognized by odorant receptors (ORs) expressed in the olfactory sensory neurons (OSNs) that consist of $\sim 1000$ members of the G-protein-coupled receptor superfamily (Touhara and Vosshall, 2009). The odorant information is directly transmitted to glomeruli in the primary olfactory center, the olfactory bulb $(\mathrm{OB})$, and further to the higher brain areas wherein odor perception is constructed (Zou et al., 2009). Because each olfactory neuron expresses a single type of OR, and the neurons expressing the same OR converge their axons onto the same glomeruli (Mombaerts, 2004), it has long been believed that glomerular response patterns faithfully reflect the pharmacological characteristics of ORs in the olfactory epithelium, and thus, odorant-OR matrices obtained by in vitro receptor assays.

Emerging evidence, however, suggests that more complex processes are involved in odorant recognition than previously appreciated. When comparing in vitro and in vivo OR responses, OR-defined glomeruli exhibited different response properties from corresponding ORs in heterologous expression systems or isolated olfactory neurons in terms of both sensitivity and speci-

Received May 17, 2010; revised Aug. 26, 2010; accepted Aug. 28, 2010.

This work was supported in part by grants from the Ministry of Education, Science, Sports, and Culture in Japan. We thank Minmin Luo for valuable discussion and Touhara laboratory members for support.

Correspondence should be addressed to Kazushige Touhara, Department of Applied Biological Chemistry, The University of Tokyo, 1-1-1 Yayoi, Bunkyo-ku, Tokyo 113-8675, Japan. E-mail: ktouhara@mail.ecc.u-tokyo.ac.jp.

DOI:10.1523/JNEUROSCI.2527-10.2010

Copyright $\odot 2010$ the authors $\quad 0270-6474 / 10 / 3016391-08 \$ 15.00 / 0$ ficity (Oka et al., 2006). Recently, the apparent higher sensitivity has been shown to be due to an efficient nasal airflow that helps absorption of odors into the mucosal layers and increases focal odor concentration in the olfactory receptor site in the mucus (Oka et al., 2009).

With respect to specificity, we previously found that some mouse ORs recognizing vanillin or acetyl isoeugenol showed different ligand specificities in HEK293 cells in vitro and in the corresponding glomerulus in vivo (Oka et al., 2006). In addition, elimination of the nasal mucus from the nasal cavity resulted in a similar change in the specificity of these glomeruli in vivo (Oka et al., 2006). These observations suggest that nasal mucus is one of the key factors in the regulation of in vivo odorant selectivity in the OB.

The nasal mucosa contains a variety of proteins such as odorant-binding proteins and metabolic enzymes (Débat et al., 2007; Mayer et al., 2009). These proteins have been thought to play a role in transporting (Tegoni et al., 2000; Pelosi, 2001; Ko et al., 2010) or decomposing or clearing (Lazard et al., 1991; BenArie et al., 1993; Miyawaki et al., 1996; Tamura et al., 1997) external volatile odorants. Deciphering the roles of such prereceptor events occurring in the mucus would lead to a better understanding of the specificity differences of ORs in vitro and in vivo, as well as of relevant odorant recognition mechanisms. This information may also be useful in answering the intriguing question regarding how these steps affect our odor perception under physiological conditions.

The purpose of this study was to determine whether nasal mucus has enzymatic activity that transforms or converts odor- 
ants to other compounds, and, if such conversion occurs, to examine whether the conversion of odorants in the mucus affects glomerular activation patterns and finally odor perception. Our study provides new insights into the role of the nasal mucus in the olfactory system.

\section{Materials and Methods}

Odorants and animals. Odorants were purchased from Tokyo Chemical Industry. Odorant solutions for analysis of enzymatic activity were prepared as $1 \mathrm{~m}$ stocks in DMSO (Wako) and then diluted with Ringer's solution to give the indicated concentrations before each experiment.

C57BL/6Cr male mice (Japan SLC) were housed in clear plastic cages. The housing room was maintained at a constant temperature $\left(24 \pm 1^{\circ} \mathrm{C}\right)$ and was on a $12: 12 \mathrm{~h}$ light:dark cycle. Mice were provided with water ad libitum. All experiments were performed in accordance with the guidelines approved by the Animal Experiments Committee of our university.

Preparation of the nasal mucus and analysis of enzymatic activity. Mice were anesthetized with a mixture of ketamine $(90 \mathrm{mg} / \mathrm{kg})$ and xylazine $(10 \mathrm{mg} / \mathrm{kg})$. Ringer's solution $(\sim 10 \mu \mathrm{l})$ was perfused into the nasal cavity at once using a shortened gel-loading tip (Ringer's solution containing the following (in $\mathrm{mm}$ ): $140 \mathrm{NaCl}$, $5.6 \mathrm{KCl}, 10.0 \mathrm{HEPES}, 2.0$ pyruvic acid sodium salt, $1.25 \mathrm{KH}_{2} \mathrm{PO}_{4}, 2.0 \mathrm{MgCl}_{2}, 2.0 \mathrm{CaCl}_{2}, 9.4$ glucose) at a depth of $12 \mathrm{~mm}$ in nasal cavity. To reduce contamination of respiratory mucus, mice were laid on their back and the tip was inserted in the dorsal area of nasal cavity. The nasal lavage fluid was then immediately collected using the same tip by pipetting the solution in-and-out. We defined this fluid as nasal mucus. Ten microliters of an odorant solution was added to $10 \mu$ l of nasal mucus, and the mixture was incubated for $5 \mathrm{~min}$ at $37^{\circ} \mathrm{C}$. The compounds were extracted from the mixture by ethyl acetate.

Chromatographic conditions of GC/MS analysis. Gas chromatographymass spectrometry (GC/MS) (GCMS-QP2010, Shimazu) was used to examine the ethyl acetate extract. The compounds were separated using a Stabilwax column $(60 \mathrm{~m} \times 0.32 \mathrm{~mm}$ inner diameter, film thickness $=0.5$ $\mu \mathrm{m})$. The column temperature was held at $40^{\circ} \mathrm{C}$ for $1 \mathrm{~min}$, then programmed to rise to $100^{\circ} \mathrm{C}$ at $10^{\circ} \mathrm{C} / \mathrm{min}$ and then to $220^{\circ} \mathrm{C}$ at $5^{\circ} \mathrm{C} / \mathrm{min}$, and finally held at this temperature for $20 \mathrm{~min}$. The interface temperature was maintained at $250^{\circ} \mathrm{C}$, and the ion source temperature was set at $230^{\circ} \mathrm{C}$. Peak identities were confirmed by matching the component mass spectra to the National Institute of Standards and Technology Mass Spectral Database and by matching the retention time and mass spectra of the peaks to the data observed for the pure compounds. To identify the products of enzymatic reaction in the nasal mucus, mass spectra were obtained in full scan mode. Quantitative analysis of products was performed by comparison with the peak area of authentic odorants in total ion chromatograms (TIC). The amount of benzoic acid was quantified by the extracted ion chromatograms, $m / z 122$. The conversion ratio was calculated by subtracting the amount of substrate remaining after conversion from the amount of starting material and then dividing by the amount of starting material. Because the quantities of compounds involved were very small, the odorants applied into the nasal cavity in a vapor phase were analyzed by the GC/MS-selected ion monitoring (SIM) process.

$\mathrm{Ca}^{2+}$ imaging in the $\mathrm{OB}$. For $\mathrm{Ca}^{2+}$ imaging, we loaded dextranconjugated calcium dye into OSNs as described previously (Oka et al.,
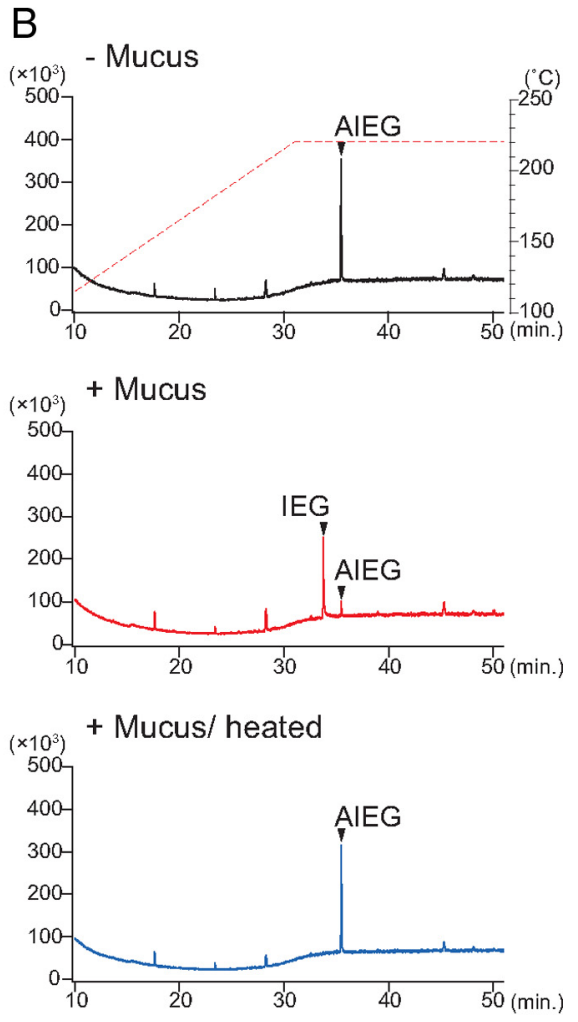

. chromatograms of the organic extracts from Ringer's solution (black line, top panel), nasal mucus (red line, middle panel), and heated nasal mucus (blue line, bottom panel), each incubated with benzaldehyde. The extracted ion chromatogram of the molecmiddle panel), and heated nasal mucus (blue line, bottom panel), each incubated with acetyl isoeugenol (AIEG). Peaks for own by arrowheads.

2006). Briefly, OSNs of 4-week-old C57BL/6Cr mice were loaded in vivo with Calcium Green-1 dextran (Invitrogen). Six microliters of dye solution $\left(6 \%\right.$, in $\mathrm{Ca}^{2+}$-free Ringer's solution) was perfused into the nasal cavity using a shortened gel-loading tip under ketamine $(90 \mathrm{mg} / \mathrm{kg})$ and xylazine $(10 \mathrm{mg} / \mathrm{kg})$ anesthesia. Maintaining body temperature at $37^{\circ} \mathrm{C}$, mice recovered from anesthesia were held for $4 \mathrm{~d}$ before imaging to allow transport of dye to OSN axon terminals.

Mice were anesthetized by intraperitoneal injection of ketamine (70 $\mathrm{mg} / \mathrm{kg}$ ) and medetomidine $(0.5 \mathrm{mg} / \mathrm{kg})$. Under local anesthesia ( $1 \% \mathrm{Xy}-$ locaine), the skin overlying the dorsal skull was removed. Using a dental drill, the bone overlying the dorsal surface of the OB was thinned and removed before imaging. Next, $1.5 \%$ agarose gel was applied and then a coverslip placed over the exposed area. An excitation wavelength of $500 \pm 25 \mathrm{~nm}$ was used for fluorescent $\mathrm{Ca}^{2+}$ imaging in the OB. Emitted light at wavelengths above $530 \mathrm{~nm}$ was collected using a $525 \mathrm{~nm}$ dichroic mirror. Stimulations were delivered by placing odorant-containing glass vial container for $3 \mathrm{~s}$ at a distance of $5 \mathrm{~mm}$ from the nose. Images were acquired using AQUA COSMOS 2.5 software and an ORCA2-ER CCD camera (Hamamatsu Photonics) at $12 \mathrm{~Hz}$ using Olympus LMPlanFL N $10 \times$ objective lens (numerical aperture $=0.25$, working distance $=21.0$ $\mathrm{mm}$, field number $=26.5 \mathrm{~mm}$ ) (Olympus). The imaging area was $2.3 \times$ $1.4 \mathrm{~mm}$. The mice were breathing freely during all experiments.

Glomerular activation patterns to an odorant were observed reproducibly in several trials, and the intensities of responses of each glomerulus in several trials were similar (supplemental Fig. S1, available at www. jneurosci.org as supplemental material).

Data analysis for imaging. The relative signals acquired from $\mathrm{Ca}^{2+}$ imaging were calculated as described previously (Wachowiak and Cohen, 2001). This method gives a measure of the signal amplitude relative to the resting fluorescence. Spatial maps of the $\mathrm{Ca}^{2+}$ imaging 
signals were constructed by subtracting the temporal average of a 2 s time window just preceding stimulus onset from a $2 \mathrm{~s}$ temporal average beginning just after odorant onset. For display, spatial maps were smoothed using a $3 \times 3$ pixel kernel. All spatial data were analyzed and processed using Aqua Cosmos 2.5 software and Photoshop (Adobe).

Preparation and nasal administration of inhibitors. Inhibitor solutions were prepared as 10,100, and $1000 \mathrm{~mm}$ stocks in DMSO and diluted to give the indicated concentrations before each experiment. For inhibiting enzymatic activity of the nasal mucus in vivo, $20 \mu \mathrm{l}$ of inhibitor solution (10 mM) was gently applied to each nostril of mice under ketamine (90 $\mathrm{mg} / \mathrm{kg})$ and xylazine $(10 \mathrm{mg} / \mathrm{kg})$ anesthesia using a shortened needle (4 $\mathrm{mm}, 29$ gauge).

Olfactory discrimination test. Four days before training, experimentally naive 6-week-old mice were placed on a food-restricted schedule. They were given 3 g per mouse $(\sim 150 \mathrm{~g} / \mathrm{kg})$ of food and trained to associate one of the two related odorants with a sugar reward for 4 consecutive days. The odorant was presented on filter paper $(25 \mathrm{~mm}$ in diameter, Whatman) placed on the bottom of a plastic Petri dish. Ten holes were drilled in the top of the Petri dish to allow the odorant to escape. During the training, the mice received four $10 \mathrm{~min}$ trials per day: two trials for an odor paired with a sugar reward and two for the unpaired odor. After $4 \mathrm{~d}$ of training, bis( $p$-nitrophenyl)phosphate (BNPP) or acetazolamide (AZ) was applied to the nasal cavities of the mice. On day 5, each test odorant was buried independently, without sugar, in the cage $(22.5 \times$ $34 \times 15.5 \mathrm{~cm}$ ) bedding at a depth of $4 \mathrm{~cm}$. The mouse behavior was recorded with a digital video camera for the analysis. The time (in seconds) spent investigating for each odorant was measured during the 2 min test period with a stopwatch. The following parametric statistics were used to look for differences in investigation time: paired $t$ test used for two related samples (pairs of odorants in each group); non-repeatedmeasures ANOVA for three independent groups (control, AZ, BNPP group).

\section{Results}

\section{Analysis of enzymatic activity of the nasal mucus}

We first determined whether the nasal mucus contained any activity that targeted odorants with various functional groups. Nasal mucus was collected as described in the Materials and Methods section. Most ORs are activated at the micromolar range according to in vitro studies (Katada et al., 2005; Oka et al., 2006), and thus the local odorant concentration in nasal mucus in vivo must be at micromolar ranges. Therefore, odorants were added at a concentration of $50 \mu \mathrm{M}$. Benzaldehyde and acetyl isoeugenol served as our initial substrates. Benzaldehyde has been known to show high variability in activation patterns in the olfactory bulb, while acetyl isoeugenol has been known to show some differences in in vitro and in vivo receptor activities (Oka et al., 2006), suggesting that these substances are likely targets of enzymatic reactions in the mucus. After $5 \mathrm{~min}$ incubation, GC/MS was used to examine whether the added odorant was intact or converted to other compounds. The TIC of samples showed that the peak for benzaldehyde in the nasal mucus was much smaller than that in a control solution, while peaks for benzyl alcohol and benzoic acid were observed (Fig. 1A). The quantification of the peaks suggested that $\sim 80 \%$ of the benzaldehyde was converted to benzyl alcohol and benzoic acid (1:5 ratio). In the case of acetyl isoeugenol, the peak for acetyl isoeugenol in the nasal mucus was barely detected and the peak for isoeugenol appeared. It was found that $>90 \%$ of acetyl isoeugenol was converted to isoeugenol (Fig. $1 B$ ). The converted products were not detected when the odorants were added in the boiled mucus, suggesting that the reaction was enzyme mediated (Fig. $1 A, B$, bottom panels).
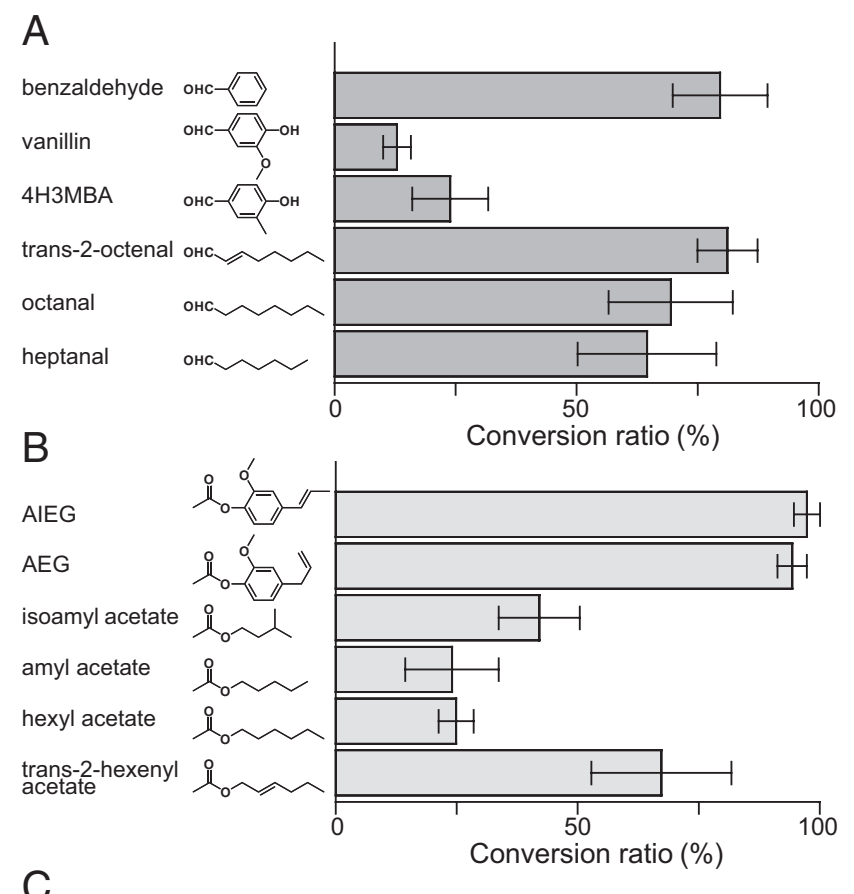

\section{No conversion}

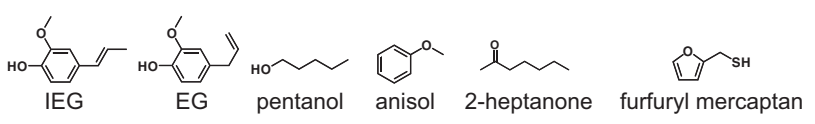

Figure 2. Structure-activity relationships of the enzyme reactions in nasal mucus of odorants with an aldehyde or acetate group. $\boldsymbol{A}$, The percentage of converted odorants with an aldehyde group (conversion ratio) was plotted as the mean \pm SEM $(n=3)$. $\boldsymbol{B}$, The percentage of converted odorants with an acetate group (conversion ratio) was plotted as the mean \pm SEM $(n=3)$. C, The compounds not converted.

\section{Structure-activity relationships of the enzyme reactions in the nasal mucus}

We next examined the structure-activity relationships of the enzymatic reactions using various aldehyde and acetate compounds. We tested aldehydes and acetates with a benzene ring (aromatic), as well as saturated or unsaturated aliphatic aldehydes and acetates. We quantified the amount of added aldehyde or acetate $(50 \mu \mathrm{M})$ remaining after 5 min incubation with nasal mucus. Figure 2 shows the percentage of converted aldehyde or acetate. Aliphatic aldehyde compounds such as octanal, heptanal, and 2-octenal were well converted in a manner similar to benzaldehyde (60-80\% conversion), whereas more substituted aromatic compounds such as vanillin and 4-hydroxy-3-methyl benzaldehyde (4H3MBA) were converted poorly in nasal mucus. Of the acetates, aromatic acetate compounds were well converted to the corresponding alcohol (90-100\% conversion), while aliphatic acetates such as isoamyl acetate, amyl acetate, and hexyl acetate were less efficiently converted in nasal mucus $(20-40 \%$ conversion). The unsaturated aliphatic acetate 2-hexenyl acetate was slightly more reactive (65\% conversion), suggesting that the presence of a double bond or conjugated structures is favorable for the reaction. These results further indicate that aldehyde and acetate are targets for enzymatic reactions. Odorants other than aldehydes and acetates, however, are not targets for enzymatic conversion in mucus: no apparent conversion has been observed for alcohol, thiol, and ketone compounds tested to date. 


\section{In vivo measurement of the} enzymatic conversions

To see whether the enzymatic conversions occur in an in vivo situation, we perfused odorant solutions directly into the nasal cavity, quickly retrieved the solution, and then analyzed the odorant conversion. In a manner similar to the reaction observed in the isolated nasal mucus, benzaldehyde was completely converted to benzyl alcohol and benzoic acid (1:4 ratio) (Fig. $3 A$ ). Acetyl isoeugenol was also completely converted to isoeugenol (Fig. 3B). Next, the odorants were applied naturally into the nasal cavity via inhalation of the compounds in the vapor phase. Again, the nasal mucus was quickly isolated for analysis. In the mucus isolated from mice that inhaled benzaldehyde, the production of benzyl alcohol and benzoic acid (2:3 ratio) was observed (Fig. 3C). In the mucus from mice that sniffed acetyl isoeugenol, the conversion to isoeugenol was observed (Fig. 3D). These results suggest that inhaled benzaldehyde and acetyl isoeugenol were enzymatically converted in the nasal mucus under in vivo conditions.

\section{Inhibitors for the enzymes that target aldehydes and acetates}

We next examined what types of enzymes convert aldehydes and acetates in nasal mucus. Various known inhibitors for aldehyde oxidase, aldehyde reductase, and aldehyde dehydrogenase were tested for the aldehyde conversion reaction.

Some reagents only partially inhibited the reaction (see Discussion). For ester hydrolysis, we tested the carboxylesterase inhibitor BNPP (Heymann and Krisch, 1967) and the carbonic anhydrase inhibitor AZ (Maren, 1952). Data were analyzed by calculating the conversion ratio and are presented by normalizing this value as a percentage of the conversion ratio without inhibitor. BNPP exhibited dose-dependent inhibition of the acetyl isoeugenol hydrolysis $\left(\mathrm{IC}_{50}=\sim 300 \mu \mathrm{M}\right)$, whereas $\mathrm{AZ}$ had little or no effect (Fig. $4 A$ ). These results suggest that the acetate hydrolysis is conducted by carboxyl esterase rather than carbonic anhydrase.

For the purpose of $\mathrm{Ca}^{2+}$ imaging, we determined whether the enzyme inhibitor BNPP stays active $1 \mathrm{~d}$ after its application in the nasal cavity. To do so, the nasal mucus was collected $1 \mathrm{~d}$ after application of BNPP and examined for enzyme activity by evaluating the acetyl isoeugenol-isoeugenol conversion. No acetyl isoeugenol was detected in the nasal mucus collected from mice treated with $\mathrm{AZ}$, whereas some remained in the mucus of BNPP-treated mice (Fig. 4B). A similar result was observed when acetyl isoeugenol was perfused into the nasal cavity (Fig. 4C). The amount of acetyl isoeugenol was quantified, and it was found that 5-20\% was left intact without conversion. These results suggest that, although there is some individual variability, BNPP is still active in vivo in the nasal cavity $1 \mathrm{~d}$ after injection.
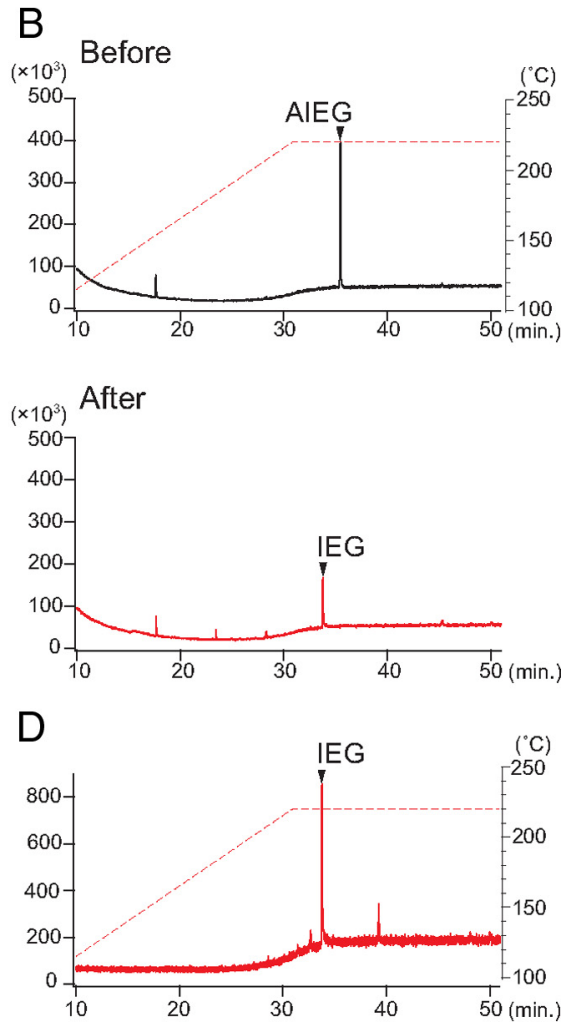

Figure 3. The in vivo enzymatic conversions of benzaldehyde and acetyl isoeugenol in the nasal cavity. $\boldsymbol{A}$, Total ion chromatogram of the benzaldehyde sample recovered from the nasal cavity. Upper panel, Control benzaldehyde; lower panel, the sample rentracted ion chromatogram of the molecular ion peak of benzoic acid $(\mathrm{m} / \mathrm{z} 122)$ is

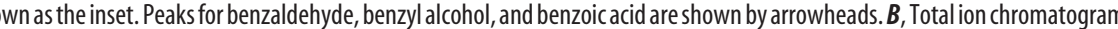
naturally inhaled benzaldehyde vapor. $\boldsymbol{D}$, Extracted ion chromatogram of the molecular ion peak $\mathrm{m} / \mathrm{z} 164$ of the nasal mucus sample obtained from mice that naturally inhaled AIEG vapor. The samples in C and $\boldsymbol{D}$ were analyzed by GC/MS-SIM.

\section{Effect of the enzymatic conversion in nasal mucus on glomerular activation patterns}

To examine the effects of the conversion in nasal mucus on the pattern of glomerular responses, we performed $\mathrm{Ca}^{2+}$ imaging of odorant responses in the olfactory bulb. We first confirmed that glomerular activation patterns to an odorant were reproducible and almost the same among several trials (supplemental Fig. S1, available at www.jneurosci.org as supplemental material). We next compared patterns of $\mathrm{Ca}^{2+}$ responses to acetyl isoeugenol before and after the application of BNPP to nasal cavities (Fig. $5 A$ ). In animal \#1, the glomeruli 2 and 3 were responsive to acetyl isoeugenol regardless of the inhibitor treatment, while the glomeruli 1 and 4 were responsive to acetyl isoeugenol only after BNPP treatment. In animal \#2, the glomeruli 2, 3, and 4 were responsive to acetyl isoeugenol regardless of the inhibitor treatment, while the glomeruli 1 and 5 were responsive to acetyl isoeugenol only after BNPP treatment. In animal \#3, although the intensity of responses of the glomeruli 1 and 2 to acetyl isoeugenol was weaker after BNPP treatment, the glomeruli 3 became strongly responsive after the treatment. These results suggest that glomerular activation patterns are affected by mucosal enzymes sensitive to BNPP. There was no effect of AZ treatment (ineffective drug as a negative control) on glomerular activation patterns (Fig. 5B). Although we are not allowed to further discuss the response profiles quantitatively due to the technical limitation of this experiment, the enzymatic conversion of acetyl isoeugenol 
A
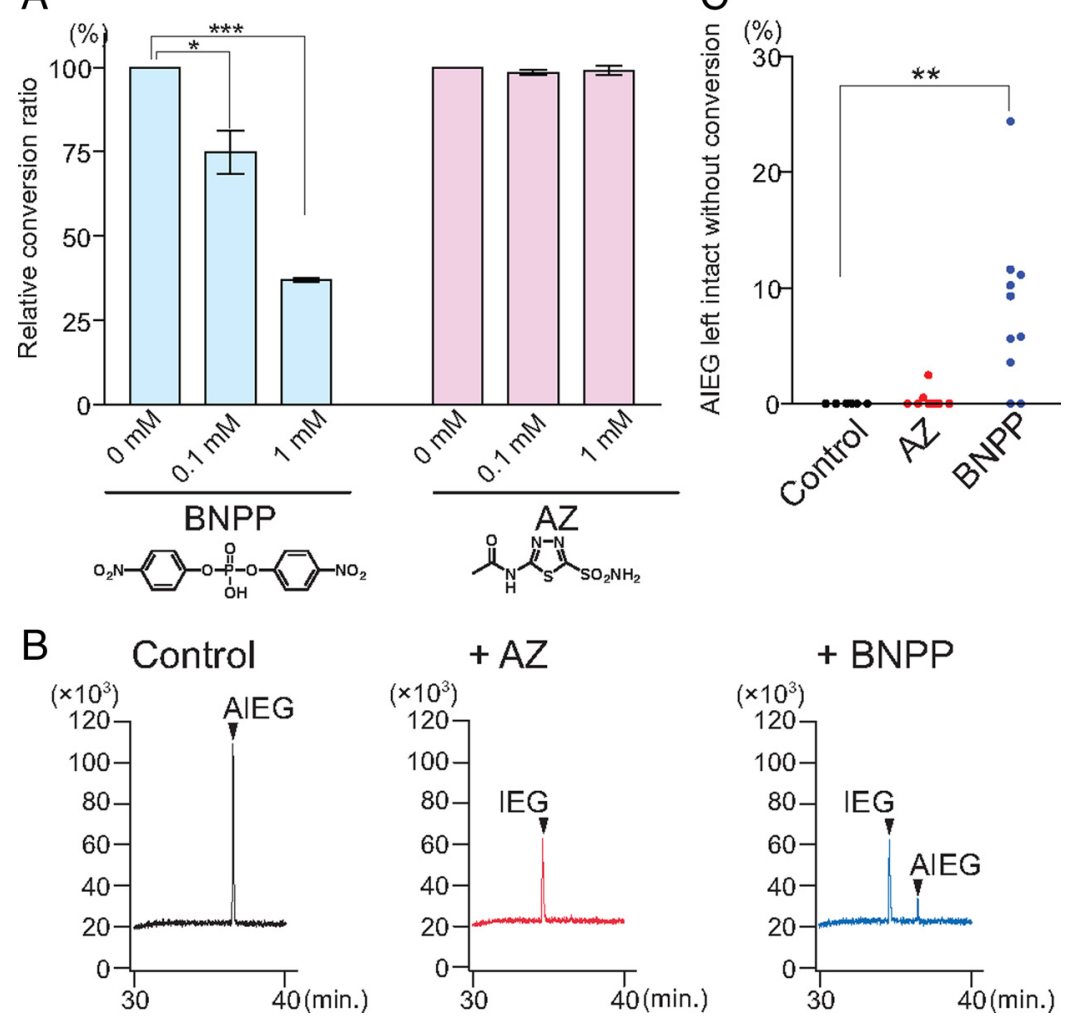

Figure 4. Effects of inhibitors for carboxylesterase and carbonic anhydrase on acetyl isoeugenol-isoeugenol conversion in nasal mucus. $A$, Dose-dependent inhibition by carboxylesterase inhibitor BNPP, but not by carbonic anhydrase inhibitor AZ. The mean conversion ratio was shown as percentage $\pm \operatorname{SEM}(n=3)$. Statistics, One-way ANOVA and post hoc test (Dunnett's test), ${ }^{*} p<$ $0.05 ;{ }^{* * *} p<0.001$. B. Analysis of the enzymatic activity of nasal mucus collected $1 \mathrm{~d}$ after application of Ringer's solution (left panel), AZ (middle panel), or BNPP (right panel) in the nasal cavity. C, The amount of nonconverted acetyl isoeugenol (AIEG) remaining $1 \mathrm{~d}$ after application of AZ or BNPP upon odorant perfusion into the nasal cavity. Statistics, One-way ANOVA and post hoc test (Dunnett's test), ${ }^{* *} p<0.01$.

seems to be fast enough to affect recognition of this odorant at the level of the olfactory sensory neuron.

\section{Effects of the enzymatic conversion on the recognition of acetyl isoeugenol in a behavioral context}

To examine the effects of the enzymatic conversion on the odor recognition at the behavioral level, we performed an olfactory discrimination test (Fig. 6). Food-restricted mice were trained to associate either of the two odorants to a sugar reward. After $4 \mathrm{~d}$ of training, the inhibitors were applied in the nasal cavities of the trained mice, and on day 5, investigation times for the two odorants were compared.

For a set of odorants that were not targets for enzyme conversion, such as eugenol and pentanol, we could successfully train the mice to discriminate the sugar-associated odorant from another odorant regardless of the presence or absence of the inhibitors (Fig. 6A). Thus, mice spent more time with a sugarassociated odorant (i.e., eugenol in Fig. $6 \mathrm{~A}$ left panel, and pentanol in Fig. $6 A$ right panel). Next, we performed the test with an odor that was not converted (eugenol in Fig. $6 B-1$, pentanol in Fig. $6 B$-2,2-heptanone in Fig. $6 B$-3) versus acetyl isoeugenol that is a target for enzyme conversion (Fig. $6 B$ ). Mice trained with a not-converted odor could discriminate it even with inhibitor treatment (Fig. 6B, left panels). When mice were trained with acetyl isoeugenol, the control or AZ-treated mice could discriminate the sugar-associated acetyl isoeugenol, while mice treated with BNPP spent significantly less time investigating acetyl isoeugenol (Fig. $6 B$, right panels), suggesting that these mice thought its odor was different from that of the acetyl isoeugenol used in their training.

If this reaction is due to conversion of acetyl isoeugenol to isoeugenol (the product of enzymatic conversion) in the mucus, mice would be confused when a sugar reward was associated with the combination of acetyl isoeugenol and isoeugenol. Indeed, mice trained with acetyl isoeugenol could not discriminate acetyl isoeugenol from isoeugenol after BNPP treatment, and thus, the mice spent an equal amount of time investigating isoeugenol and acetyl isoeugenol (Fig. 6C). These results suggest that the enzymatic conversion of acetyl isoeugenol to isoeugenol in the mucus affects the odor quality for mice at the level of perception.

\section{Discussion}

The olfactory system possesses a sophisticated neural circuitry for decoding odorant information such that the combination of activated ORs from a repertoire of thousands is represented as an odor map in the olfactory bulb. The activated receptor repertoire appears to be determined by the pharmacology of each OR (Kajiya et al., 2001; Katada et al., 2005), and therefore, it has been thought that the odorant-OR matrices obtained by in vitro ligand-receptor pairing experiments would reveal the entire view of in vivo odor coding maps. In this study, we demonstrated that metabolic enzymes in the nasal mucus immediately attack odorants that enter from the nasal cavity space, leading to a decrease in the amount of the original odorant and the formation of metabolites in the mucus before binding to the receptors. We propose that, because of this enzyme-mediated biotransformation process, mice were not decoding information for the original odorant but sensing the mixture of the original odorant and metabolites. Therefore, in vitro odor receptor matrices sometimes do not reflect the receptor code for each odorant in vivo.

In general, the role of mucus is to protect the epithelium or body surface exposed to external environments against dryness. For example, a decrease of mucus secretion causes disorders like dry eye (Paulsen, 2006) and xerostomia (dryness of the mouth) (Thelin et al., 2008). Other roles include protection against infection by microorganisms or damage by harmful chemical compounds. The mucus, therefore, contains various xenobiotic metabolic enzymes (Débat et al., 2007). The nasal mucus is not the exception; the olfactory epithelium as well as nasal and Bowman's glands secrete various metabolic enzymes such as cytochrome P450, dehydrogenases, oxidases, reductases, and esterases that degrade various chemicals coming from the outside world (Bogdanffy et al., 1987; Thornton-Manning et al., 1997; Kimoto et al., 2004; Kurosaki et al., 2004; Mayer et al., 2009). It has been demonstrated that xenobiotic metabolic reactions occur inside of mucosal cells, but it was not clear whether they occurred in the mucus. In this study, we have shown that enzyme- 
A

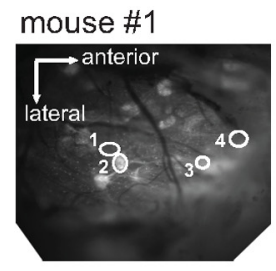

mouse \#2

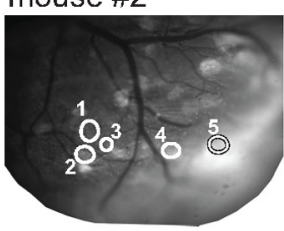

Before BNPP application

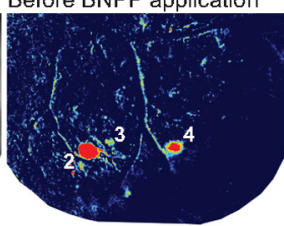

After BNPP application

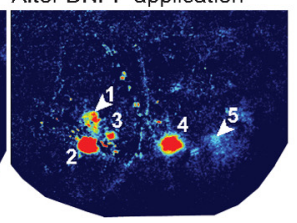

Before BNPP application
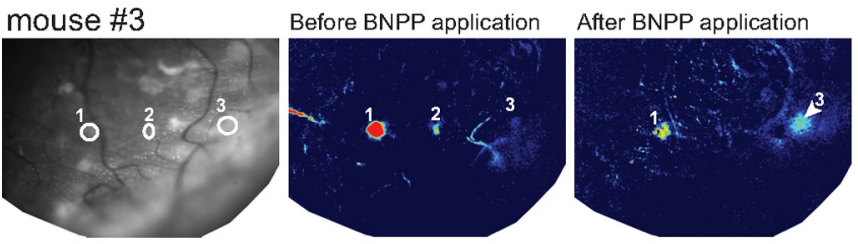

B
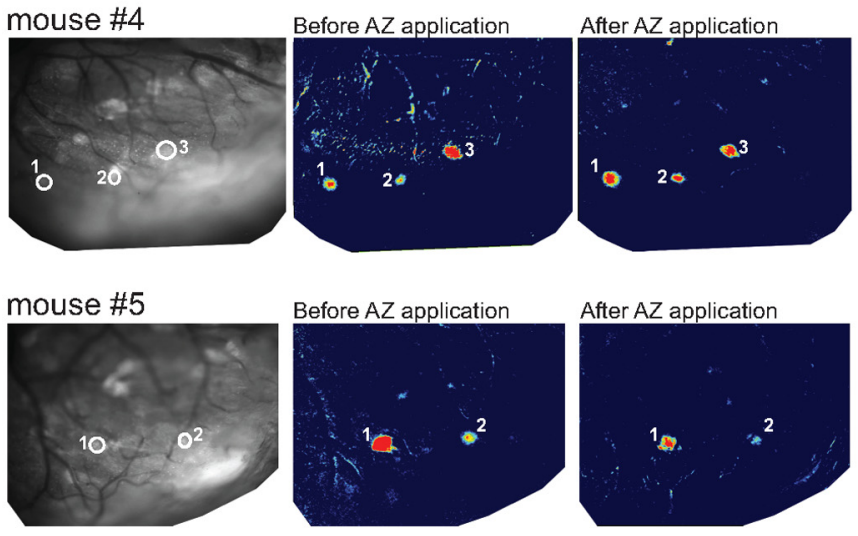
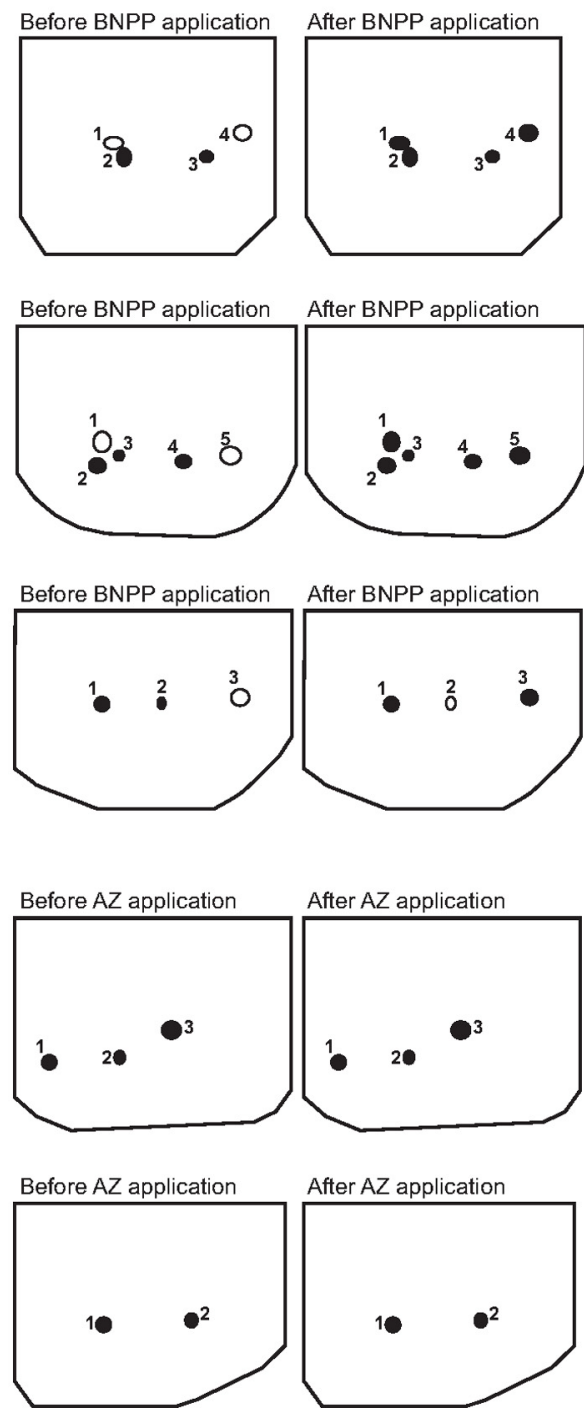

Figure 5. Comparison of odorant-evoked glomerular activation patterns stimulated with acetyl isoeugenol before and after the application of inhibitor. $A$, Glomerular Ca ${ }^{2+}$ responses to acetyl isoeugenol (AIEG) before/after the application of BNPP shown as pseudocolored images. Left, Fluorescent image of the olfactory bulb. Middle images, Pseudocolored images of the olfactory bulb before and after the application of BNPP showing the glomerular activation patterns; red corresponds to the greatest response. The data from three animals (\#1, \#2, \#3) are shown. Right, Schematic illustrations of glomerular activation patterns in pseudocolored images. The filled circles indicate glomeruli activated by AIEG, while the glomeruli with open circles are the ones that responded to AIEG only after BNPP application. Some extra glomeruli are activated by AIEG after BNPP treatment. B, Glomerular Ca ${ }^{2+}$ responses to acetyl isoeugenol (AIEG) before/after the application of AZ shown as pseudocolored images. Left, Fluorescent image of the olfactory bulb. Middle images, Pseudocolored images of the olfactory bulb before and after the application of AZ showing the glomerular activation patterns. The data from two animals (\#4,\#5) are shown. Right, Schematic illustrations of glomerular activation patterns in pseudocolored images. The filled circles indicate glomeruli activated by AIEG. There was no effect of AZ treatment on glomerular activation patterns.

mediated conversion of odorants does indeed occur in the nasal mucus, and also that these reactions appear to affect olfactory recognition at the level of olfactory sensory neurons as well as olfactory perception at the behavioral level.

Because olfactory neurons expressing the same olfactory receptors converge onto fixed glomeruli, it has been believed that the odorant-OR matrices obtained from in vitro receptor characterization exactly reflect the activation patterns in the olfactory bulb. However, the ligand specificity of an OR expressed in heterologous systems in vitro is sometimes different from that in the olfactory bulb (Oka et al., 2006). In the optical imaging experiments, it has been empirically noticed that bulbar activation patterns of some odorants such as heptyl aldehyde and benzaldehyde vary significantly among multiple trials even in the same individual (Fried et al., 2002). In these cases, consistent bulbar activation patterns are only observed after washing the nasal cavity with Ringer's solution (Oka et al., 2006). Thus, these observations can be explained by the presence of metabolizing enzymes and by the difference in the conversion efficiency in nasal mucus at each recording condition.

The amount of secreted proteins in the nasal mucus may depend on the metabolic or reproductive status of the individual. For example, OBP-1F secretion is shown to be downregulated following food deprivation (Badonnel et al., 2007). Similarly, it is possible that the amount of odorant metabolizing enzymes in nasal mucus may vary depending on physiological conditions. Also, age-related progression of lesions has been observed in the Bowman's gland in olfactory mucosa (Kondo et al., 2009), which may affect the amount of mucosal enzymes. The expression levels of these enzymes may be different between races, as was found for 
A

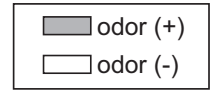

$E G(+)$ vs. pentanol(-)

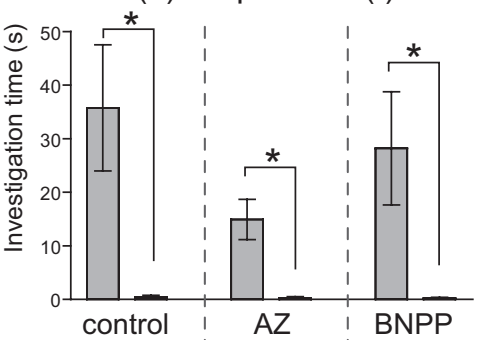

B-1 $E G(+)$ vs. AlEG(-)

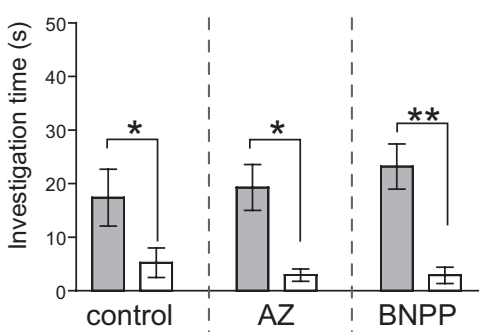

B-2 pentanol(+) vs. AIEG(-)

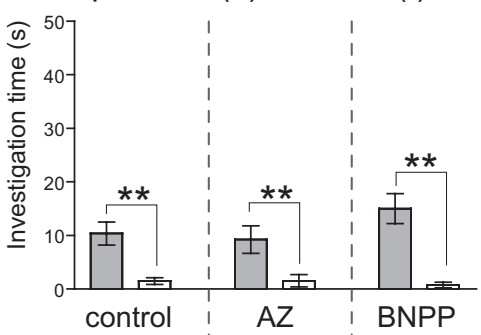

B-3 2-heptanone(+) vs. AlEG(-)

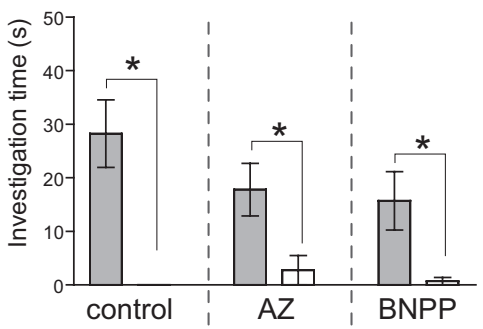

C IEG(+) vs. AlEG(-)

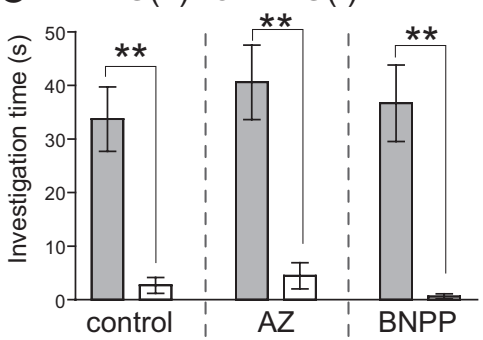

EG(-) vs. pentanol(+)

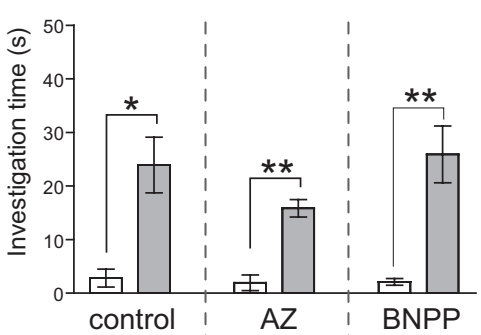

$E G(-)$ vs. AlEG(+)

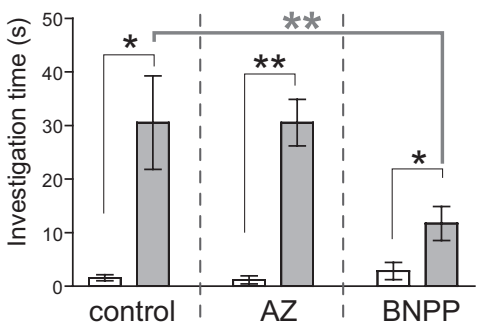

pentanol(-) vs. AlEG(+)

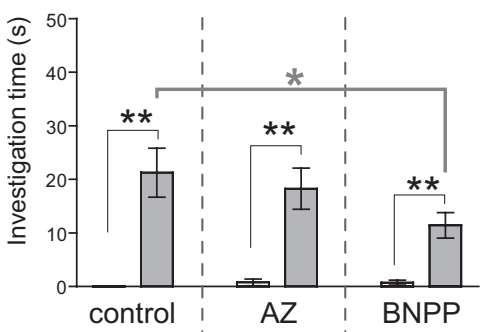

2-heptanone(-) vs. AlEG(+)

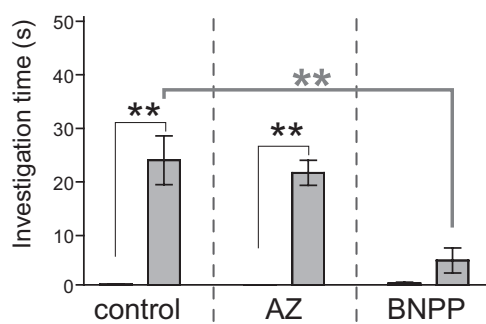

IEG(-) vs. AlEG(+)

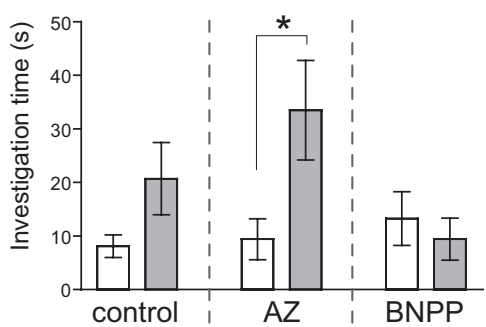

Figure 6. Effects of enzymatic conversion on the recognition of acetyl isoeugenol (AIEG) in a behavioral context. Mice were trained for $4 \mathrm{~d}$ to associate a reward (sugar grains) with either of two odorants. After $4 \mathrm{~d}$ of training, BNPP or AZ was applied to the nasal cavities of the mice. On day 5 , investigation times were measured for each pair of odorants without the sugar reward. Mean investigation times (in seconds) \pm SEM during the 2 min test period are shown as bar graphs for odorants paired with the sugar reward [odor (+), gray bars], and unpaired odorants [odor (-), white bars]. $\boldsymbol{A}$, Eugenol (EG) versus pentanol; $\boldsymbol{B}-1$, EG versus AIEG;

aldehyde dehydrogenase (Chen et al., 1991). The difference in olfactory perception to the same odorant under different hormonal conditions (Doty and Cameron, 2009; Prud'homme et al., 2009), along with aging (Ship and Weiffenbach, 1993), or between races (Doty et al., 1985; Jones et al., 1995) may be due to differences in the composition of the mucosal components. The unraveled role of the nasal mucus as a place for odorant biotransformation may shed light on indirect effects of an endocrine condition on odor perception, and thus may provide a tool to monitor physiological change in each individual.

In this study, we found that, in nasal mucus, aldehydes were converted to alcohols and acids. To determine which enzyme is involved in these reactions, we tested various inhibitors. Aldehyde oxidase inhibitors such as SKF525A and benzamidine did not inhibit the reaction, while an aldehyde reductase inhibitor, 3,5-dichlorosalicylic acid, partially inhibited the acid formation, and aldehyde dehydrogenase inhibitors such as cyanamide and citral also exhibited only partial inhibitory activity. Although both aldehyde dehydrogenase and oxidase have been shown to be secreted from Bowman's glands in the olfactory epithelium (Kurosaki et al., 2004; Débat et al., 2007), the type of enzymes that act as metabolic enzymes for aldehyde odorants remains to be determined. For the ester conversion, our results suggest that carboxyl esterase, but not carbonic anhydrase, is likely involved in the reaction. Consistently, it has been reported that carboxyl esterase activity in the olfactory mucosa is much higher than that in the respiratory mucosa (Bogdanffy et al., 1999).

The nasal mucus has also been implicated in the process for dissolving hydrophobic odorant molecules, transporting them to receptor sites, and finally degrading and removing the excess odorants. Indeed, the olfactory mucus contains socalled odorant binding proteins that bind odorant molecules. Odorant-odorant binding protein complexes have been reported to be internalized into sustentacular cells (Brose et al., 2009). In addition,

$\boldsymbol{B}$-2, pentanol versus AIEG; $\boldsymbol{B}$-3, 2-heptanone versus AIEG; $\boldsymbol{C}$ IEG versus AIEG. Statistics, Paired $t$ test was used for two related samples (pair of odorants in each group); ${ }^{*} p<0.05$, ${ }^{* *} p<0.01$ (black asterisk). Non-repeated-measures ANOVA for three independent groups (control, AZ, BNPP group) and post hoc test (Dunnett's test), ${ }^{*} p<0.05,{ }^{* *} p<0.01$ (gray asterisk). 
the sustentacular cells express organic anion transporters that take up carbonic acids from the mucus (Monte et al., 2004; Kaler et al., 2006). This activity could explain our observation that the amount of benzoic acid found in the nasal cavity in vivo upon benzaldehyde inhalation is much less than that found in in vitro experiments using isolated mucus. It may also explain why some aliphatic alcohols were recovered in only minute quantities from the nasal cavity. The nasal mucus thus appears to play a critical role not only in efficiently absorbing odorants from the air space in the nasal cavity, but also in removing them to prepare for the next odor stimuli.

In conclusion, we have demonstrated that mucosal enzymes metabolize odorants with an aldehyde or acetate functional group, and as a consequence, mice perceive the odorant as a mixture of the original odorant and its metabolites. The effect of prereceptor enzymatic events in the mucus on odor perception is likely a common "epiphenomenon" among mammals. Indeed, a recent study in humans demonstrated that inhibitors of human nasal enzymes influence the quality of odorants (Schilling et al., 2007). The current study proposes the intriguing notion that our olfactory sense is sometimes deceived because of the mucus.

\section{References}

Badonnel K, Denis JB, Caillol M, Monnerie R, Piumi F, Potier MC, Salesse R, Baly C (2007) Transcription profile analysis reveals that OBP-1F mRNA is downregulated in the olfactory mucosa following food deprivation. Chem Senses 32:697-710.

Ben-Arie N, Khen M, Lancet D (1993) Glutathione S-transferases in rat olfactory epithelium: purification, molecular properties and odorant biotransformation. Biochem J 292:379-384.

Bogdanffy MS, Randall HW, Morgan KT (1987) Biochemical quantitation and histochemical localization of carboxylesterase in the nasal passages of the Fischer-344 rat and B6C3F1 mouse. Toxicol Appl Pharmacol 88:183-194.

Bogdanffy MS, Manning LA, Sarangapani R (1999) High-affinity nasal extraction of vinyl acetate vapor is carboxylesterase dependent. Inhal Toxicol 11:927-941.

Brose H, Strotmann J, Breer H (2009) List of abstracts from the XVIIIth Congress of European Chemoreception Research Organization, ECRO2008: uptake of odorant binding proteins in the olfactory epithelium. Chem Senses 34:E58.

Chen CC, Hwu HG, Yeh EK, Morimoto K, Otsuki S (1991) Aldehyde dehydrogenase deficiency, flush patterns and prevalence of alcoholism: an interethnic comparison. Acta Med Okayama 45:409-416.

Débat H, Eloit C, Blon F, Sarazin B, Henry C, Huet JC, Trotier D, Pernollet JC (2007) Identification of human olfactory cleft mucus proteins using proteomic analysis. J Proteome Res 6:1985-1996.

Doty RL, Cameron EL (2009) Sex differences and reproductive hormone influences on human odor perception. Physiol Behav 97:213-228.

Doty RL, Applebaum S, Zusho H, Settle RG (1985) Sex differences in odor identification ability: a cross-cultural analysis. Neuropsychologia 23:667-672.

Fried HU, Fuss SH, Korsching SI (2002) Selective imaging of presynaptic activity in the mouse olfactory bulb shows concentration and structure dependence of odor responses in identified glomeruli. Proc Natl Acad Sci U S A 99:3222-3227.

Heymann E, Krisch K (1967) [Phosphoric acid-bis-(p-nitro-phenylester), a new inhibitor of microsomal carboxylesterases]. Hoppe Seylers Z Physiol Chem 348:609-619.

Jones RE, Brown CC, Ship JA (1995) Odor identification in young and elderly African-Americans and Caucasians. Spec Care Dentist 15:138-143.

Kajiya K, Inaki K, Tanaka M, Haga T, Kataoka H, Touhara K (2001) Molecular bases of odor discrimination: reconstitution of olfactory receptors that recognize overlapping sets of odorants. J Neurosci 21:6018-6025.

Kaler G, Truong DM, Sweeney DE, Logan DW, Nagle M, Wu W, Eraly SA, Nigam SK (2006) Olfactory mucosa-expressed organic anion transporter, Oat6, manifests high affinity interactions with odorant organic anions. Biochem Biophys Res Commun 351:872-876.

Katada S, Hirokawa T, Oka Y, Suwa M, Touhara K (2005) Structural basis for a broad but selective ligand spectrum of a mouse olfactory receptor: mapping the odorant-binding site. J Neurosci 25:1806-1815.

Kimoto M, Iwai S, Maeda T, Yura Y, Fernley RT, Ogawa Y (2004) Carbonic anhydrase VI in the mouse nasal gland. J Histochem Cytochem 52:1057-1062.

Ko HJ, Lee SH, Oh EH, Park TH (2010) Specificity of odorant-binding proteins: a factor influencing the sensitivity of olfactory receptor-based biosensors. Bioprocess Biosyst Eng 33:55-62.

Kondo K, Watanabe K, Sakamoto T, Suzukawa K, Nibu K, Kaga K, Yamasoba T (2009) Distribution and severity of spontaneous lesions in the neuroepithelium and Bowman's glands in mouse olfactory mucosa: age-related progression. Cell Tissue Res 335:489-503.

Kurosaki M, Terao M, Barzago MM, Bastone A, Bernardinello D, Salmona M, Garattini E (2004) The aldehyde oxidase gene cluster in mice and rats. Aldehyde oxidase homologue 3, a novel member of the molybdoflavoenzyme family with selective expression in the olfactory mucosa. J Biol Chem 279:50482-50498.

Lazard D, Zupko K, Poria Y, Nef P, Lazarovits J, Horn S, Khen M, Lancet D (1991) Odorant signal termination by olfactory UDP glucuronosyl transferase. Nature 349:790-793.

Maren TH (1952) Pharmacological and renal effects of diamox (6063), a new carbonic anhydrase inhibitor. Trans N Y Acad Sci 15:53.

Mayer U, Küller A, Daiber PC, Neudorf I, Warnken U, Schnölzer M, Frings S, Möhrlen F (2009) The proteome of rat olfactory sensory cilia. Proteomics 9:322-334.

Miyawaki A, Homma H, Tamura H, Matsui M, Mikoshiba K (1996) Zonal distribution of sulfotransferase for phenol in olfactory sustentacular cells. EMBO J 15:2050-2055.

Mombaerts P (2004) Genes and ligands for odorant, vomeronasal and taste receptors. Nat Rev Neurosci 5:263-278.

Monte JC, Nagle MA, Eraly SA, Nigam SK (2004) Identification of a novel murine organic anion transporter family member, OAT6, expressed in olfactory mucosa. Biochem Biophys Res Commun 323:429-436.

Oka Y, Katada S, Omura M, Suwa M, Yoshihara Y, Touhara K (2006) Odorant receptor map in the mouse olfactory bulb: in vivo sensitivity and specificity of receptor-defined glomeruli. Neuron 52:857-869.

Oka Y, Takai Y, Touhara K (2009) Nasal airflow rate affects the sensitivity and pattern of glomerular odorant responses in the mouse olfactory bulb. J Neurosci 29:12070-12078.

Paulsen F (2006) Cell and molecular biology of human lacrimal gland and nasolacrimal duct mucins. Int Rev Cytol 249:229-279.

Pelosi P (2001) The role of perireceptor events in vertebrate olfaction. Cell Mol Life Sci 58:503-509.

Prud'homme MJ, Lacroix MC, Badonnel K, Gougis S, Baly C, Salesse R, Caillol M (2009) Nutritional status modulates behavioural and olfactory bulb Fos responses to isoamyl acetate or food odour in rats: roles of orexins and leptin. Neuroscience 162:1287-1298.

Schilling B, Gfeller H, Koch H, Granier T, Ding X, Locher E (2007) List of abstracts from the Twenty-Ninth Annual Meeting of the Association for Chemoreception Sciences: inhibitors of nasal enzymes influence the perceived quality of odorants. Chem Senses 32:125.

Ship JA, Weiffenbach JM (1993) Age, gender, medical treatment, and medication effects on smell identification. J Gerontol 48:M26-32.

Tamura H, Miyawaki A, Inoh N, Harada Y, Mikoshiba K, Matsui M (1997) High sulfotransferase activity for phenolic aromatic odorants present in the mouse olfactory organ. Chem Biol Interact 104:1-9.

Tegoni M, Pelosi P, Vincent F, Spinelli S, Campanacci V, Grolli S, Ramoni R, Cambillau C (2000) Mammalian odorant binding proteins. Biochim Biophys Acta 1482:229-240.

Thelin WR, Brennan MT, Lockhart PB, Singh ML, Fox PC, Papas AS, Boucher RC (2008) The oral mucosa as a therapeutic target for xerostomia. Oral Dis 14:683-689.

Thornton-Manning JR, Nikula KJ, Hotchkiss JA, Avila KJ, Rohrbacher KD, Ding X, Dahl AR (1997) Nasal cytochrome P450 2A: identification, regional localization, and metabolic activity toward hexamethylphosphoramide, a known nasal carcinogen. Toxicol Appl Pharmacol 142:22-30.

Touhara K, Vosshall LB (2009) Sensing odorants and pheromones with chemosensory receptors. Annu Rev Physiol 71:307-332.

Wachowiak M, Cohen LB (2001) Representation of odorants by receptor neuron input to the mouse olfactory bulb. Neuron 32:723-735.

Zou DJ, Chesler A, Firestein S (2009) How the olfactory bulb got its glomeruli: a just so story? Nat Rev Neurosci 10:611-618. 